\title{
Acerca de números, dificultades e intervenciones
}

\author{
Sandra Torresi ${ }^{1,2 \star}$ \\ 1 Universidad Favaloro; informes@favaloro.edu.ar \\ 22Universidad de la Marina Mercante; info@udemm.edu.ar
}

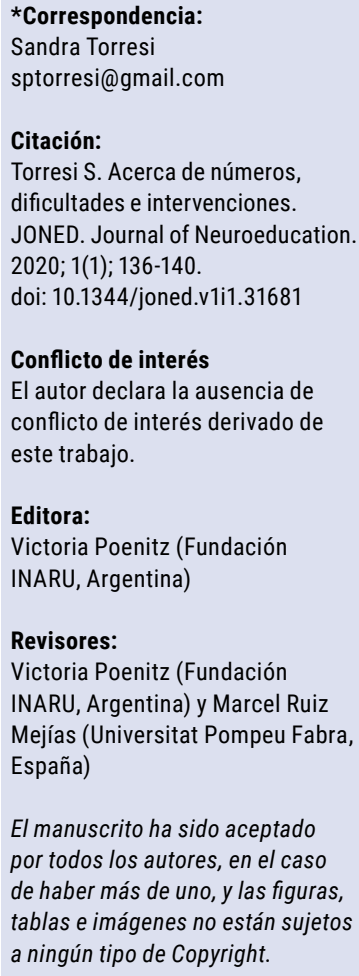

\section{Resumen}

La capacidad para utilizar las representaciones simbólicas en diferentes contextos es un recurso adaptativo indispensable para resolver con fluidez las situaciones numéricas. El debate científico acerca de los procesos cognitivos específicos implicados en el desarrollo de las habilidades numéricas se ha intensificado considerablemente en los últimos años y sus conclusiones podrían impactar en futuras conceptualizaciones. De cualquier manera, los aportes actuales de la neurociencia y la psicología cognitiva se traducen en un marco referencial interdisciplinario disponible para diferenciar las dificultades en el aprendizaje matemático y la discalculia de desarrollo, diseñar intervenciones preventivas y proponer prácticas educativas adecuadas a los perfiles de desempeño en el dominio de lo numérico.

\begin{abstract}
The ability to use symbolic representations in different contexts is an essential, adaptive resource to solve mathematical problems with ease. In recent times, a debate has opened in science about the specific cognitive processes involved in numerical competence which could impact future conceptualizations regarding the characteristics of mathematical learning difficulties. However, the current contributions from neuroscience and cognitive psychology are translated into an interdisciplinary frame of reference which describes mathematical learning difficulties and developmental dyscalculia, designing preventive interventions and proposing educational practices suitable to students' performance profiles.
\end{abstract}

Palabras clave: sentido numérico, sistema de aproximación numérica, discalculia, dificultad de aprendizaje matemático, modelo de respuesta a la intervención 


\section{Introducción}

La capacidad para utilizar representaciones numéricas simbólicas es un pilar cultural imprescindible para desenvolverse adaptativamente en el entorno social: contar dinero, decir la hora, comprender la relación distancia/tiempo y resolver situaciones cotidianas en las que están involucrados los números. En los últimos años, la neurociencia cognitiva, la neuropsicología, la psicología cognitiva y educación comenzaron a orientar sus investigaciones hacia la identificación de los procesos nucleares de la cognición numérica, las dificultades en el desarrollo del aprendizaje del número y los abordajes educativos $^{1-3}$. Los aportes interdisciplinarios disponibles, aunque en pleno debate y provisorios, permiten intervenir preventivamente desde un modelo basado en la evidencia científica y diferenciar los perfiles de desempeño de las dificultades de origen multicausal y la dificultad que deriva de un déficit específico en el desarrollo de lo numérico.

\section{Acerca del número...}

Desde el momento del nacimiento, disponemos de recursos adaptativos para extraer información del entorno: mecanismos cognitivos que nos permiten distinguir los colores, orientarnos en el espacio ${ }^{4}$ y ver el mundo numéricamente. Para estimar la numerosidad de una colección como propiedad de los objetos contamos con un sentido numérico innato y anterior al lenguaje ${ }^{5,6}$. Esta capacidad intuitiva responde a un componente cognitivo central arraigado en nuestra historia evolutiva y ontogenética llamado sistema de aproximación numérica (ANS). El ANS se activa, con mayor o menor precisión, ante tareas de comparación de colecciones a lo largo de toda la vida, desde la infancia hasta la adultez $z^{7,9}$.

La posición teórica dominante acerca del origen de las habilidades aritméticas vincula al sentido numérico preverbal con el sentido numérico verbal y simbólico que se desarrolla posteriormente ${ }^{8}$. La precisión del sistema de aproximación numérica (ANS) se considera un precursor de la adquisición de la matemática formal simbólica y predictor estable de las habilidades numéricas ${ }^{9}$. Sin embargo, revisiones muy recientes revelan inconsistencias entre la precisión del ANS y el desempeño matemático que desafían científicamente las hipótesis vigentes. El debate se plantea sobre diferentes aspectos de este proceso nuclear: sus características distintivas, la extensión de su base neurobiológica, la forma de medir su precisión y su relación con el sentido numérico simbólico ${ }^{10}$.

En adelante, el consenso científico acerca de los procesos específicos será un aporte clave para la comprensión del desarrollo típico de la competencia numérica y de sus dificultades. Mientras tanto, el corpus de conocimiento entre teoría, investigación y práctica que está disponible, si bien provisorio y en permanente reformulación, conforma un marco de referencia que colabora en la identificación de las dificultades en el aprendizaje del número y el cálculo y en el diseño de intervenciones.

\section{Acerca del número y las dificultades...}

En un grupo de estudiantes con indicadores de desempeño aritmético bajo, es probable que muchos tengan dificultades en el aprendizaje matemático (DAM) y que solamente algunos tengan discalculia del desarrollo (DD). ¿Cómo se diferencian?

Varios componentes interactúan en el aprendizaje aritmético, el contexto, la matemática como disciplina, la didáctica y los recursos de quien aprende: motivación, afectividad, procesos cognitivos generales y procesos cognitivos específicos del dominio numérico. Una de estas dimensiones o una combinación de ellas puede constituirse en un obstáculo para el desarrollo de esta competencia.

La dificultad en el aprendizaje matemático (DAM) es multicausal, por lo que el bajo desempeño en tareas aritméticas puede explicarse por un déficit en alguno de los procesos cognitivos generales (atención, memoria de trabajo, lenguaje, procesamiento visoespacial, autorregulación), por ansiedad matemática, fobia escolar, motivación, causas pedagógicas o inclusive por más de una variable simultáneamente. La dificultad es secundaria a otra condición y no están comprometidos los procesos específicos del dominio numérico. Por lo tanto, el desempeño en matemática mejora significativamente cuando se interviene sobre la dimensión o el aspecto que obstaculiza su desarrollo ${ }^{10}$.

En cambio, la discalculia del desarrollo (DD) es una dificultad específica del aprendizaje en los aspectos más básicos del procesamiento de número y el cálculo por un déficit en el sentido numérico y en 
la representación de cantidad ${ }^{11-14}$. Si bien en la literatura científica con frecuencia se usan los términos "matemática y aritmética" como sinónimos, la discalculia del desarrollo se vincula con la dimensión aritmética de la matemática y no con la geometría. Su origen es neurobiológico y presenta anomalías cerebrales estructurales y funcionales ${ }^{15}$. Las neuroimágenes de niños con DD durante la ejecución de tareas de comparación de magnitudes revelan menor densidad de materia gris respecto de los niños sin DD y menor activación en el surco intraparietal (IPS), estructura neural fundamental en la representación abstracta del número ${ }^{16}$. Se manifiesta de forma muy heterogénea con comorbilidades altas con otras dificultades del aprendizaje, alcanza un $30-40 \%$ en el caso de dislexia y del $10-20 \%$ con AD$\mathrm{HD}^{1,17}$. La discalculia del desarrollo es una dificultad primaria severa y persistente en niños con escolaridad adecuada y con desarrollo intelectual típico que impacta, no solo en el rendimiento académico, sino también en todas aquellas situaciones de la vida cotidiana en las que se necesita el uso fluido de los números ${ }^{5,18}$.

Por lo tanto, la diferenciación DAM o DD requiere una evaluación general de recursos intelectuales, lenguaje y procesos lectores, memoria de trabajo y funciones ejecutivas y, además, una evaluación de los procesos específicos. El sentido numérico se activa ante tareas de comparación de cantidades no simbólicas: ¿hay más puntos azules o amarillos?, comparación de cantidades simbólicas: ¿cuál es mayor: 83 ?, velocidad de procesamiento (tiempos de ejecución), fluidez de cálculos simples de un dígito: $4+3$ y ubicación de números en una recta. La información acerca del nivel cognitivo se integra a los restantes niveles de análisis del desarrollo, comportamental, afectivo, neurobiológico y contextual, para construir un perfil singular de fortalezas y debilidades en el aprendizaje matemático 5,9,19,20. Finalmente, ya sea que los estudiantes tengan DAM o DD, la pregunta que guía todas las acciones es ¿cómo los ayudamos?

\section{Acerca del número, las dificultades y las intervenciones...}

La escuela es el espacio en donde los procesos nucleares de la cognición numérica se desarrollan en forma sistemática. Por lo tanto, es allí en donde se debe detectar tempranamente a los estudiantes con dificultades en el desarrollo del número y el cálculo (Tabla 1) e intervenir en su trayectoria de aprendizaje con estrategias basadas en la evidencia.

En este sentido, los aportes científicos sobre cómo se aprende se traducen en principios y estrategias de enseñanza que benefician a todos los estudiantes en general y especialmente a los que tienen alguna dificultad en las habilidades numéricas ${ }^{5,21-27}$ :

- optar por una metodología de enseñanza explícita, sistemática y cíclica

- determinar el nivel de desarrollo de los procesos específicos (línea de base) para diseñar secuencias didácticas

- focalizar en los conceptos nucleares: subitización, conteo y aproximación, asociación representación verbal-representación numérica

- focalizar en el desarrollo de las funciones ejecutivas: control inhibitorio, memoria de trabajo y flexibilidad cognitiva

- favorecer el "poner en palabras": qué hicieron y por qué (habilidades metacognitivas)

- seguir la secuencia concreto-figurativo-simbólico

- focalizar en la resolución de situaciones de la vida cotidiana

- contextualizar las situaciones a resolver y representarlas gráficamente

- segmentar procedimientos complejos en sus componentes

- evitar la ejercitación reproductiva sin reflexión

- usar un lenguaje muy claro y puntual

- considerar el impacto emocional de las dificultades de aprendizaje: estrés, ansiedad, fobia, baja autoestima

- acompañar la identificación del error y promover la autocorrección

- crear un ambiente con estímulos novedosos evitando distractores

- utilizar recursos tecnológicos para dinamizar las propuestas y recibir feedback inmediato sobre tiempos de respuesta, aciertos y errores

En los últimos años ha tomado relevancia el modelo de respuesta a la intervención (RTI), un abordaje preventivo multinivel que se basa en la identificación de indicadores de riesgo de dificultad de aprendizaje académico y la implementación de un sistema de intervención con validez empírica y niveles de inten- 
sidad que se van ajustando de acuerdo con la evolución de los aprendizajes del estudiante ${ }^{28}$.

\section{Conclusiones}

El debate científico es clave para la búsqueda de conocimientos acerca de la cognición numérica y sus dificultades ${ }^{7,9,24,29}$ : la capacidad predictiva del desempeño en ANS y los beneficios del entrenamiento de su precisión o la necesidad de redefinir el sentido numérico y el desarrollo de la representación simbólica ${ }^{10}$. Sin embargo, es probable que mientras esto sucede en el contexto de investigación, en una escuela, un estudiante angustiado y con bajo rendimiento académico en matemática esté esperando respuestas sobre qué le sucede, por qué y cuáles serían las intervenciones que podrían mejorar su rendimiento. Entonces, y a pesar de los debates vigentes, el desafío es traducir los aportes interdisciplinarios disponibles en recursos para ${ }^{30}$ :

a) comprender el desarrollo y el aprendizaje desde un modelo integrado de sus distintos niveles de análisis

b) identificar tempranamente las dificultades de aprendizaje

c) implementar programas de intervención preventivos y de habilitación basados en la evidencia

Le escuela es un espacio para la observación atenta de los trayectos de desarrollo típico o con dificultades, un espacio para la observación de los cambios y las transformaciones que suceden a partir de las experiencias de enseñanza... la escuela es un lugar de cuidado para todos los estudiantes.

\section{Referencias}

1. Dehaene S. The number sense: How the mind creates mathematics (revised and updated edition). New York: Oxford University Press; 2011.

2. Cohen Kadosh R, Dowker A. (Eds.). The Oxford Handbook of Numerical. Oxford: Oxford Library of Psychology; 2015.

3. Dennis MS, Sharp E, Chovanes J, Thomas A, Burns RM, Custer $B$, et al. A meta-analysis of empirical research on teaching students with mathematics learning difficulties. Learn. Disabil. Res. Pract. 2016; 31, 156-168.
Tabla 1. Indicadores de dificultad. Reporte de observaciones de clase. Educación Inicial y Primaria. Bs. As., 2017

- subitizar (cuantificación rápida y exacta de sets de 3/4 elementos sin contar)

- estimar (cuantificación aproximada de una colección de objetos)

- contar (cuantificación exacta de varios elementos): errores en la secuencia, de correspondencia, en conteo descendente

- comparar números (ordenar de menor a mayor/mayor a menor)

- reconocer patrones

- comprender reglas y símbolos

- reconocer las regularidades del sistema de numeración decimal

- transcodificar (cambiar con fluidez de una representación numérica a otra: 45 , cuarenta y cinco, /cuarentaicinco/)

- completar rectas numéricas

- recordar automáticamente tablas de multiplicar, números dobles $(3+3)$, cálculos simples $(8+1)$, complementos de $10(7+3)$

- resolver cálculos mentales simples (memoria de trabajo y descomposición numérica)

- resolver operaciones (en especial restas y divisiones)

- abstraer conceptos básicos (conmutatividad, operaciones inversas)

- comprender equivalencias del sistema monetario

- manejar magnitudes y proporciones (recordar secuencias temporales, estimar y comparar tiempo, velocidad y distancia, hora en formato analógico)

- alternar estrategias de resolución de problemas (flexibilidad cognitiva)

- organizar espacialmente cantidades (alineación y direccionalidad)

- recordar reglas y fórmulas

- comprender mapas y gráficos

- reconocer objetos en 3D desde diferentes perspectivas

- comunicar procesos matemáticos

- autorregular y auto monitorear (evaluar habilidades para resolver un problema, seleccionar estrategias, organizar la información y monitorear resultados)
4. Spelke E, Sang Ah L, Izard V. Beyond core knowledge: natural geometry. Cogn Sci. 2010; 34(5): 863-884.

5. Butterworth B. Dyscalculia. From Science to Education. NY: Routledge. 2019.

6. Xu F, Spelke ES, Goddard S. Number sense in human infants. Dev Sci. 2005; 8(1):88-101.

7. Libertus M, Odic D, Halberda J. Intuitive sense of number correlates with math scores on college-entrance examination. Acta Psychologica. 2012; 141: 373-379. 
8. Brannon EM, Jordan KE, Jones SM. Behavioral signatures of numerical discrimination. En Platt ML, Ghazanfar AA (Eds.). Primate neuroethology Oxford. Oxford: Oxford University Press; 2010. 44-159.

9. Halberda J, Mazzocco M, Feigenson L. Individual differences in non-verbal number acuity correlate with maths achievment, Nature. 2008; 455:665-668.

10. Wilkey $E$, Ansari D. Challenging the neurobiological link between number sense and symbolic numeral abilities. Ann.N.Y.Acad.Sci. 2019; 1-23.

11. Balbi A, Dansilio S. Dificultades de aprendzaje del cálculo: contribuciones al diagnóstico psicopedagógico. Cs. Ps. 2010; IV (1):7-15.

12. Wilson A, Dehane $S$. Number sense and developmental dyscalculia. En Coch D, Dawson G, Fischer KW (Eds.). Human behavior, learning, and the developing brain: Atypical development. NY: The Guilford Press. 2007 (212-238).

13. Schneider M, Beeres K, Coban L, Merz S, Schmidt S, Stricker $\mathrm{J}$, et al. Associations of non-symbolic and symbolic numerical magnitude processing with mathematical competence: A meta-analysis. DevScience. 2016; 20:1-16.

14. Piazza M, Facoetti A, Trussardi A, Berteletti I, Conte S, Lucangeli $D$. Developmental trajectory of number acuity reveals a severe impairment in developmental dyscalculia. Cognition. 2010; 116(1):33-41.

15. Molko N, Cachia A, Rivière D, Mangin J, Bruandet M, Le Bihan $D$, et al. Functional and Structural Alterations of the Intraparietal Sulcus in a Developmental Dyscalculia of Genetic Origin. Neuron. 2003; 40: 847-858.

16. Sokolowski HM, Fias W, Mousa A, Ansari D. Common and distinct brain regions in 582 both parietal and frontal cortex support symbolic and nonsymbolic number processing in 583 humans: A functional neuroimaging meta-analysis. Neuroimage. 2016. doi:10.1016/j.neuroimage.2016.10.028.

17. Szucs D, Devine A, Soltesz F, Nobes A, Gabriel F. Developmental dyscalculia is related to visuo-spatial memory and inhibition impairment. Cortex. 2013; 49: 2674-88.

18. Andersson, U. Skill development in different components of arithmetic tests of executive functioning in children. Child Neuropsychology. 2010; 5: 115- 129. doi: 10.1076/ chin.5.2.115.3167.
19. Libertus ME, Odic D, Halberda J. Intuitive sense of number correlates with math scores on college-entrance examination. Acta Ps. 2012; 141:373-379.

20. Mejias S, Mussolin C, Rousselle L, Grégoire J, Noël M. Numerical and non-numerical estimation in children with and without mathematical learning disabilities. Child Neurop. 2011; 37-41. doi: 10.1080/09297049.2011.6253 55.

21. Gillespie A, Graham S. (2014). A Meta-Analysis of Writing Interventions for Students with Learning Disabilities. Exceptional Children. 2014; 80(4): 454-473.

22. Clements D, Sarama J, Germeroth C. Learning executive function and early mathematics: Directions of casual relations. Early Childhood Research Quarterly. 2016; 36: 79-90.

23. Feigenson L, Libertus ME, Halberda J. Links between the intuitive sense of number and formal mathematics ability. Child Dev Perspectives. 2013;7(2):74-79. doi:10.1111/ cdep.12019.

24. Xin, Jitendra, Deatline-Bunchman. The Effect of Schema-Based Instruction in Solving Mathematics Word Problems: An Emphasis on Prealgebraic Conceptualization of Multiplicative Relations. Journal for Research in Mathematics Education. 2008; 39(5):526-551.

25. Cueli M, González-Castro P, Krawec J, Núñez JC, González-Pienda JA. Hipatia: a hypermedia learning environment in mathematic. Anales de Psicología. 2016; 32(1): 98-105. doi: 10.6018/analesps.32.1.185641.

26. Carey E, Hill F, Devine A, Szücs D. The chicken or the egg? The direction of the relationship between mathematics anxiety and matthematics performance. Front Psychol. 2016; 6:.

27. Gilmore CK, McCarthy SE, Spelke ES. Non-symbolic arithmetic abilities and mathematics achievement in the first year of formal schooling. Cognition. 010;115(3):394-406. doi: 10.1016/j.cognition.2010.02.002.

28. Fuchs, D., y Fuchs, L. (2006. Introduction to response to intervention: What, why, and how valid is it? Reading Research Quarterly. 2006; 41(1): 93-99.

29. Park, K, Brannon, E. Improving arithmetic performance with number sense training: an investigation of underlying mechanism. Cognition. 2014; 133(1):188-200.

30. Loeches, M. Neuroscience and education: we already reached the tipping point. Rev Psico. 2015; 2: 67-70. 